\title{
COMMENT LES VOCATIONS PHILOLOGIQUES ÉVOLUENT À TRAVERS LES GÉNÉRATIONS
}

\section{REMARQUES PRÉLIMINAIRES}

Le monde entier est en constante évolution. Cette évolution, qui apporte des changements nécessaires, est également présente dans le monde universitaire, dans les cercles de néophilologues, et par conséquent chez les romanistes. La question n'est donc pas de s'interroger sur le bien-fondé du phénomène, mais éventuellement sur sa rapidité accrue ces dernières années.

Aussi, en interprétant le titre de ce numéro de Romanica Wratislaviensia: De la philologie romane aux études françaises - évolution ou rupture?, je penche plutôt pour l'idée d'une évolution que d'une rupture, et c'est elle qui va m'amener, dans la suite de cet article, à adopter une méthode peu répandue dans ce genre de publications. Tout en observant l'évolution des programmes et des objectifs de la formation dans ce qu'on s'est habitué à appeler, à l'université, la philologie romane, et sans oublier la dimension de la recherche, je tâcherai de l'illustrer par une expérience tout à fait personnelle, celle de ma famille, et de démontrer que les études romanes ont subi durant les soixante-dix dernières années une évolution progressive, déterminée d'une part par les différents contextes politiques de notre pays, et de l'autre, par les changements généraux survenus sur le marché économique européen ou même mondial. Je sais que dans un article destiné à une revue scientifique, on devrait se garder de toute allusion personnelle ou familiale. Mais l'histoire des trois générations de romanistes de ma famille, qui s'inscrit dans celle de quatre universités polonaises - Cracovie, Łódź, Varsovie et Poznań — illustre 
d'une certaine manière les grands tournants des quatre-vingt dernières années. Du reste, cette présentation à caractère très personnel ne se prétend pas généralisable à toute la communauté des romanistes polonais.

\section{LES DÉBUTS}

Les plus anciennes philologies romanes de Pologne sont celles des universités Jagellonne, de Varsovie et de Poznań. Toutes se sont développées progressivement, grâce à des professeurs qui avaient le don de former des enseignants et d'organiser autour d'eux des groupes de chercheurs et d'étudiants intéressés par les langues et littératures romanes. Le mot romanes a son importance, car dans toutes ces universités, traditionnellement, il ne s'agissait pas seulement de la langue française, mais aussi de l'italien, de l'espagnol, du portugais et même parfois du roumain (à Cracovie, à Poznań). Ainsi peut-on lire dans l'information préparée à l'occasion du $125^{\mathrm{e}}$ anniversaire des études romanes de Cracovie (2017) ${ }^{1}$ que dans l'entre-deux-guerres, celles-ci attiraient beaucoup de monde entre autres parce qu'elles proposaient des cours d'autres langues romanes : italien, espagnol et roumain. Les cultures romanes étaient à l'honneur tant dans les recherches que dans les cours des enseignants, lesquels n'étaient pas tous forcément romanistes de formation.

La deuxième guerre mondiale a entrainé la fermeture des universités, mais elles ont continué de fonctionner dans la clandestinité. Leurs activités, forcément réduites sous la contrainte, ont demandé une réorganisation des formes d'enseignement. Et c'est à ce moment que commence, à Cracovie, l'histoire de ma famille de philologues. L'Université Jagellonne est du reste caractéristique, en ce sens qu'elle est aussi la première en Pologne à avoir proposé des études romanes.

\section{LA GUERRE}

La Seconde Guerre mondiale a donc été une période difficile pour la romanistique cracovienne. À cette époque, il n'y avait que trois romanistes à Cracovie, Maria Malkiewicz-Strzałkowa, Zbigniew Gierczyński et le lecteur Henri Bernard². Les études romanes ne se sont cependant pas interrompues dans les années 1942 1945. C'est ainsi que, après avoir passé son baccalauréat au lycée clandestin à

${ }^{1}$ URL : <http://www.ifr.filg.uj.edu.pl/documents/1586406/135345378/125-ROMANISTYKA1. pdf/f1348815-9f43-45df-af12-76f2ff4b1884> [consulté le 15.02.2018].

2 Ibidem. 
Cracovie le 18 juin $1943^{3}$, ma mère Władysława Roszczynowa, née Gowin ${ }^{4}$, a pu entreprendre en septembre 1943 des études romanes à la Faculté de Philosophie de l'Université Jagellonne Clandestine. Les cours étaient dispensés en cachette à de petits groupes, dans des maisons privées, des sacristies ou autres lieux convenus. C'est dans ce contexte difficile que ma mère a été formée suivant le modèle classique : littérature, latin, grammaire historique. Quant à sa connaissance parfaite du français parlé, elle lui venait de sa formation à l'école primaire et au collège des ursulines de Cracovie. Cet enseignement était à l'époque d'un très haut niveau et assurait une bonne connaissance de la langue. C'est également chez les ursulines qu'elle a développé sa passion pour la culture française.

\section{LES LANGUES À L'UNIVERSITÉ APRÈS 1945}

Libérée de l'occupation nazie, la Pologne rouvre à partir de 1946 ses universités et en fonde de nouvelles. De nouveaux programmes de formation se mettent en place, dans lesquels figurent les matières linguistiques et littéraires. Mais la guerre a décimé la population, et les spécialistes capables d'assurer ce type de formation sont rares. Certaines universités (Varsovie, Cracovie, Poznań, Wrocław) arrivent malgré tout à faire renaître des chaires de langues étrangères ou à proposer d'autres formes de cours de langue, comme les lectorats. Des philologies de langues étrangères voient également le jour à l'Université de Łódź nouvellement fondée.

Le $1^{\text {er }}$ octobre 1945 en effet, Bolesław Kielski a été nommé professeur au département de langue et de littérature française, ce qui a donné naissance à la philologie romane de cette université. L'année universitaire 1945-1946, elle accueille un premier groupe de 14 étudiants $^{5}$. Parmi eux, se trouve ma mère qui a dû quitter Cracovie pour des raisons familiales. Elle entre ainsi dans l'histoire des philologies romanes polonaises, car Łódź, ville ouvrière, n'avait pas précédemment de tradition universitaire. Le maigre personnel survivant de la guerre s'applique à y enseigner la littérature, la culture et la grammaire françaises, mais aussi l'espagnol. Et c'est dans ce contexte assez difficile que, le 18 novembre 1950, Władysława Roszczynowa obtient son diplôme de maîtrise en philologie française.

${ }^{3}$ Ce baccalauréat a été validé après la guerre en 1945 sous la forme d'un certificat délivré par le Lycée Królowa Wanda.

4 Toutes les informations bibliographiques à propos de ma mère proviennent tout d'abord de ma connaissance de sa vie, mais pour supporter ces données, je me réfère à la publication de Mieczysław Gajos, « Wspomnienie, Doktor Władysława Maria Insarew-Roszczynowa (1923-2013) », Neofilolog 40/1, 2013, pp. 7-14. Ce texte a aussi été publié dans Języki Obce w Szkole 105 (<www. jows.pl/node/451>) et dans Kronika Uniwersytetu Łódzkiego 3 (135), 2013, pp. 43-45.

${ }^{5}$ URL : <http://romanistyka.uni.lodz.pl/onas.htm> [consulté le 15.02.2018]. 
Encore étudiante, elle avait commencé à enseigner le français au lycée numéro XVII de Łódź, car toutes les écoles manquaient alors cruellement d'enseignants, tout en devant faire face à une nombreuse population de jeunes gens qui, pendant les cinq années de guerre, n'avaient pas pu suivre de formation régulière à tous les niveaux d'enseignement et étaient censés rattraper leurs retards en peu de temps.

Mais alors que l'on tente ainsi de ressusciter ou de réorganiser l'enseignement secondaire et universitaire des langues étrangères, voilà que survient la période stalinienne qui met à l'arrêt presque tous les départements de langues occidentales, dont le français, ainsi que l'enseignement de ces langues à l'école. Les départements et chaires de langues de l'université entrent ainsi dans une phase d'hibernation qui se caractérise par une certaine clandestinité des chercheurs qui, dans la majorité des cas, se voient interdire d'enseigner leurs matières. Cette situation dramatique frappe aussi les écoles primaires et secondaires, et beaucoup de diplômés des philologies, enseignants de langues, doivent donc chercher du travail ailleurs. Tel est également le sort de ma mère, qui en 1950, à la suite d'une directive de la Commission nationale du travail (Państwowa Komisja Przydziału Pracy), est réaffectée dans un bureau d'import-export de l'industrie du papier.

En 1956, tout le bloc communiste connaît un nouveau tournant historique que l'on a appelé le « dégel ». Dans la foulée, l'on procède à la réactivation de certaines philologies occidentales, mais avec un nombre restreint d'étudiants. Dès le début, le ministère impose son modèle de programme de formation des philologues, obligatoire dans toutes les universités quelle que soit la langue enseignée. Mais le dégel autorise aussi la réintroduction des langues occidentales dans les écoles secondaires, et cela permet à ma mère de revenir à l'école en 1959. C'est là qu'elle va développer son intérêt, tout d'abord pratique puis scientifique, pour la didactique des langues.

\section{LA NAISSANCE DE LA LINGUISTIQUE APPLIQUÉE}

Fondateur puis directeur dans les années 1965-1976 du Département de linguistique appliquée de l'Université de Poznań, Ludwik Zabrocki apporte une nouveauté dans les néophilologies polonaises. Dans son activité et ses recherches, il met l'accent sur l'aspect pratique de la linguistique, et notamment sur un enseignement pratique des langues étrangères.

Parallèlement, ma mère enseigne successivement dans deux lycées de Łódź : le numéro XXII, et à partir de 1965, le numéro XIII. C'est dans ce dernier qu'elle introduit dans l'enseignement pratique du français la nouvelle méthode audio-visuelle qu'elle a découverte à l'occasion de plusieurs stages en France. C'est ainsi que de philologue, elle se transforme en véritable enseignante qui n'appuie plus son enseignement sur la compréhension et la traduction de textes littéraires, 
mais cherche à enseigner un français de communication. La recherche aussi a évolué dans ce sens, ce qui amène ma mère à préparer, hors du cadre universitaire, une thèse de doctorat basée sur son expérience en milieu scolaire. Elle l'écrit sous la direction du professeur Zabrocki. Soutenue en 1970 à l'Université Adam Mickiewicz, la thèse s'intitule Zastosowanie metody audiowizualnej "Voix et images de France " w szkole średniej ogólnoksztatcacej [L'introduction de la méthode audiovisuelle "Voix et images de France » à l'école secondaire]. Elle obtient le titre de docteur en linguistique appliquée, et c'est un nouveau tournant dans l'histoire des études romanes polonaises, car voici qu'une enseignante de français devient docteur non pas en romanistique mais en linguistique appliquée.

L'idée du professeur Zabrocki est continuée par son disciple, le professeur Franciszek Grucza. Avec quelques collaborateurs, il fonde en 1972 l'Institut de linguistique appliquée de l'Université de Varsovie. Cet établissement développe la recherche en didactique des langues et en traductologie, mais introduit aussi des programmes de formation en traduction et interprétation qui seront à l'origine de l'École supérieure des langues (WSJO), qui deviendra plus tard l'Institut de linguistique appliquée. Ces nouveaux programmes orientés vers l'enseignement des langues et la traduction s'écartent significativement de la formation des philologies traditionnelles de l'époque par une diminution des matières ayant trait aux études littéraires au profit de matières à caractère plus professionnel. Cependant, ils doivent toujours intégrer certains éléments de la formation philologique obligatoire dans toutes les universités polonaises. Ils forment, dirons-nous, une sorte de passerelle entre l'ancien et le nouveau.

L'exemple de la linguistique appliquée prendra encore davantage de poids tout à l'heure, à l'appui de mes réflexions sur la vision des changements de la philologie romane dans la lignée de son évolution.

\section{LES ANNÉES SOIXANTE-DIX}

Revenons à l'exemple de la philologie romane de l'Université de Łódź Après son « hibernation » qui durait vingt ans (1950-1971), à la rentrée 19711972, on a réintroduit son fonctionnement et on a recruté les premiers étudiants. Son directeur de l'époque, le professeur Kazimierz Kupisz ne pensait pas encore qu'on puisse introduire dans les programmes des matières ayant trait à la linguistique appliquée et à la formation à un métier déterminé.

C'est à ce moment que ma mère intègre cet établissement, pour y assurer d'une part l'enseignement de la grammaire historique, et de l'autre la méthodologie de

${ }^{6}$ Les données présentées dans la suite proviennent de mon savoir personnel mais aussi du site web de l'Institut d'Études romanes de l'Université de Łódź (Historia Romanistyki Uniwersytetu Łódzkiego) : <http://romanistyka.uni.lodz.pl/onas.htm>. 
l'enseignement du français. Elle incarne personnellement le passage entre la définition ancienne de la philologie et les exigences d'une formation nouvelle. Grâce à ses compétences, à partir de 1973, les étudiants de Łódź se voient proposer un nouveau séminaire de maîtrise en méthodologie de l'enseignement du français.

C'est à cette étape de la saga familiale que j'entre en scène. Je viens de terminer mes études secondaires au lycée IV de Łódź, un lycée à programme d'anglais renforcé. Mais je décide de suivre la tradition familiale, et j'entreprends des études de philologie romane à l'Université de Łódź. Mes deux premières années suivent le programme universitaire imposé par le ministère. Mais en deuxième année, j'ai appris l'existence du nouveau programme mis en place à l'Institut de linguistique appliquée de Varsovie. Peu désireuse de poursuivre des études « à l'ancienne », je me présente à l'examen d'entrée de cette nouvelle formation, je passe le test d'aptitude linguistique, et je peux ainsi commencer ces études qui sont une nouveauté à l'époque. Mais lorsque je les terminerai, le diplôme que j'obtiendrai me distinguera à peine des autres romanistes, signalant simplement les nouvelles spécialisations en traduction et didactique des langues ${ }^{7}$. Je suis ensuite engagée en tant qu'assistante à la philologie romane de Łódź, en 1979.

\section{LES ANNÉES QUATRE-VINGT}

Comme le remarquent Magdalena Dybaś, Agnieszka Dziemianowicz-Bąk, Magdalena Krawczyk-Radwan et DominikaWalczak, « [En Pologne] avant 1989, nous avions affaire à un nombre limité d'écoles supérieures, à une structure transparente de l'enseignement universitaire, à des contenus de formation standardisés, et en outre, les écoles supérieures dépendaient d'un budget centralisé et ne connaissaient pas de concurrence $»[\operatorname{trad} . \mathrm{T} \text {. T. }]^{8}$.

Les contenus d'enseignement des départements de français, en effet, ne variaient pas d'une philologie à l'autre. Tous les étudiants suivaient un programme incluant des matières littéraires, linguistiques, un apprentissage intensif de la langue française et d'autres langues romanes. Mais aussi, de plus en plus fréquemment, la méthodologie de l'enseignement des langues étrangères. À quelques exceptions près, les études de l'époque se concentraient sur la littérature et la civilisation françaises sans prendre en compte les autres dimensions de la francophonie. La traductologie et la formation professionnelle des traducteurs et interprètes restaient absentes des programmes. Qui plus est, nous avons vécu au même

\footnotetext{
7 «Magister filologii romańskiej, specjalność thumaczeniowa oraz specjalność nauczycielska ».

${ }^{8}$ M. Dybaś, A. Dziemianowicz-Bąk, M. Krawczyk-Radwan, D. Walczak, « Szkolnictwo wyższe », <RoSE2011-R3-szkolnictwo-wyzsze-2.pdf > [consulté le 5.09.2017] : « [W Polsce] przed 1989 rokiem mieliśmy raczej do czynienia z niewielką liczbą uczelni, przejrzystością struktury, jednakowymi treściami kształcenia, a także $\mathrm{z}$ wolnym od konkurencji centralnie finansowanym szkolnictwem wyższym ».
} 
moment, et pendant quelques années, une expérience du ministère qui a raccourci les études de 5 à 4 ans. Cette initiative a apporté de nombreux bouleversements qui, heureusement n'ont pas duré longtemps.

Il faut toutefois souligner que pendant les années soixante-dix et quatre-vingt, les études de langues, y compris de français, étaient très demandées. Le nombre de places soumis au principe de numerus clausus était toujours nettement inférieur au nombre de candidats.

\section{LA RÉVOLUTION DE 1989}

La période suivante s'ouvre en 1989 , c'est-à-dire après la chute du communisme en Pologne. À partir de ce moment, des changements considérables se manifestent dans tous les domaines : juridique, administratif, social, éducatif, culturel. Nous pouvons dire qu'au début des années quatre-vingt-dix, les universités connaissent une véritable révolution. C'est également vrai pour les facultés et départements de langues vivantes, ce qui s'exprime, entre autres, par une pression sociale qui réclame des changements de programmes d'enseignement afin d'apporter aux étudiants des compétences de communication, mais aussi en traduction, dans un large éventail de langues étrangères autres que le russe. Cette révolution, pourtant, ne s'est pas faite du jour au lendemain. Il a fallu tout d'abord former du personnel enseignant, développer la recherche, et attendre le développement d'une nouvelle politique éditoriale et d'un nouveau marché de la traduction.

En réponse à cette demande, on voit naître dans tout le pays beaucoup de nouvelles écoles supérieures (państwowe szkoły wyższe, PSW) et d'autres établissements privés où s'ouvrent des départements de langues proposant eux aussi une formation en traduction, car celle-ci apparaît comme un symbole de nouveauté et de liberté. Mais, dans cette prolifération d'offres du début des années quatrevingt-dix, rares étaient les milieux qui avaient déjà une certaine expérience de la formation des traducteurs et qui pouvaient la développer.

Cette nouvelle vague touche aussi les départements de français. On essaie de moderniser les programmes en répondant en quelque sorte à la demande des étudiants. Ainsi n'est-il pas possible de parler de rupture, mais plutôt et certainement d'une lente évolution de l'image du romaniste. Elle est d'autant plus nécessaire qu'une nouvelle concurrence se manifeste pour les philologies traditionnelles, avec l'apparition de collèges de formation de maîtres de langues étrangères ( $\mathrm{na}$ uczycielskie kolegia języków obcych, NKJO) créés tout spécialement pour préparer de futurs enseignants, ce qui avait toujours été précédemment la vocation des philologies romanes.

C'est dans ce contexte que se situe mon expérience personnelle. J'ai obtenu mon doctorat en 1986, avec une thèse basée sur un corpus traductologique mais 
relevant toujours davantage de la linguistique que de l'approche comparative. À l'occasion de ma thèse d'habilitation, en revanche, je serai l'une des premières à aborder la question de la traduction audiovisuelle suivant une approche méthodologique encore naissante en Europe : l'analyse conversationnelle. Mon travail est remarqué dans les milieux académiques occidentaux ${ }^{9}$, et ce, à un moment où certains collègues romanistes polonais ne jugent pas encore ce sujet digne d'intérêt. Mes recherches dans ce domaine permettront pourtant au professeur Józef Sypnicki, directeur de la philologie romane de Poznań où j'ai travaillé à partir de 1984, d'ouvrir un nouveau département de traductologie dont je suis devenue directrice.

Je suis ainsi, après ma mère, une deuxième romaniste polonaise à s'être trouvé une nouvelle vocation en suivant une voie dictée par les attentes de son époque : elle, dans la formation des enseignants de langues étrangères, et moi, dans la formation de chercheurs pour une nouvelle spécialité : la traductologie.

\section{LES PREMIÈRES RÉGULATIONS}

Une des premières lois de l'enseignement universitaire de l'après-1989 a été votée le 12 septembre $1990^{10}$. Cette loi attribuant aux universités une plus grande autonomie, entre autres, en matière de programmes d'enseignement, a sans doute marqué un certain tournant dans la formation des romanistes, puisque chaque institut ou chaire de philologie romane pouvait désormais concevoir ses propres programmes d'enseignement. Mais elle n'a pas apporté de véritable révolution, car une bonne partie des professeurs, attachés à leur tradition, ne voulaient pas renoncer à leurs matières et à leur forme d'enseignement « à l'ancienne ». Les nouvelles libertés se heurtent parfois à l'inertie de ceux qui les reçoivent.

Or, cette inertie des romanistes intervient à un moment où l'évolution de la société leur impose de nouvelles formes de concurrence :

- multiplication anarchique des établissements d'enseignement privés qui incluent dans leur offre une formation en langues,

- création des NKJO qui se développent dans de nombreuses villes sans tradition académique,

${ }^{9}$ Dans la bibliographie d'une publication de J. Ivarsson et M. Caroll, Subtitling, TransEdit HB, Simirshamn 1998, figure une référence à ma thèse d'habilitation à côté de laquelle on peut lire : "Very valuable studies on the processes of condensation, deletion, paraphrase, etc. » (p. 170). Cette remarque élogieuse me semble d'autant plus importante que ma thèse, publiée aux éditions de notre université, ne pouvait à l'époque bénéficier d'une ample diffusion.

${ }^{10}$ Loi relative à l'enseignement supérieur du 12 septembre 1990. 
- montée en puissance de l'anglais, désormais reconnu comme seule langue de communication internationale.

Les milieux romanistes n'avaient probablement pas prévu ces obstacles.

\section{LA FONDATION DE L'ASSOCIATION ACADÉMIQUE DES ROMANISTES POLONAIS «PLEJADA»}

Avant les années 1990, les philologies romanes recevaient des aides et subventions de l'Ambassade de France à Varsovie. Celle-ci organisait des rencontres des directeurs de départements de français et avait son mot à dire dans leurs politiques de formation, car elle distribuait des bourses aux étudiants et aux chercheurs, elle subventionnait des rencontres scientifiques, et parfois, des publications.

Or, la France, étant membre de l'UE depuis bien plus longtemps que la Pologne, avait compris que la structure des recherches en « romanistique » devait être redirigée vers de nouvelles voies tenant compte des enjeux de l'internationalisation et l'interdisciplinarité. C'est dans ce sens que, dès janvier 1993, le conseiller universitaire de l'Ambassade de France m'a demandé d'organiser un colloque intitulé Langage et Travail. L'intention était d'ouvrir les romanistes aux études pluridisciplinaires. D'autres initiatives, par exemple la création de formation en français pour les juristes ou les économistes, ont également été lancées.

$\mathrm{Vu}$ les obstacles mentionnés tout à l'heure, la situation des romanistes polonais au début des années 90 n'était pas encourageante, et l'on savait très bien que l'Ambassade de France ne soutiendrait pas infiniment la formation et la recherche des philologies romanes polonaises. À la fin des années 1990, M. Olivier Jacquot, alors attaché de coopération pour le français à l'Ambassade de France de Varsovie, a lancé l'idée de fonder une association de tous les romanistes universitaires, dans la lignée de l'association des romanistes tchèques, Gallica ${ }^{11}$, fondée en 2000. C'est ainsi que l'Association académique des romanistes polonais « Plejada » est née en 2002. J'ai eu la chance et l'honneur d'en assumer les fonctions de secrétaire générale (2002-2006) et de présidente (2006-2010). Et cette association a joué par la suite un rôle majeur dans les différentes phases de l'évolution des études romanes ${ }^{12}$.

11 Pour de plus amples informations concernant la fondation de " Gallica » et la coopération entre les romanistes tchèques et polonais, voir entre autres l'article d'O. Pešek, « L'Association Gallica fête son dixième anniversaire », Écho des études romanes, Vol. VI/Num. 1-2, 2010, pp. 233-236.

12 Pour plus d'informations concernant l'activité de « Plejada », voir T. Tomaszkiewicz, T. Giermak-Zielińska, T. Jaroszewska, « Dix années de l'Association académique des romanistes polonais PLEJADA », [dans :] Les études françaises dans les pays de Visegrád, T. Jaroszewska (dir.), Oficyna Wydawnicza LEKSEM, Łask 2012, pp. 13-20. 


\section{L'ATRP «PLEJADA» ET SON IMPACT SUR LA FORME DE LA ROMANISTIQUE POLONAISE}

« Plejada » s'est engagée dès le début des années 2000 dans le débat sur l'avenir de la philologie romane traditionnelle. Les défis que les philologies romanes ont dû affronter à partir du début de ce siècle étaient les suivants :

— introduction du processus de Bologne par la Loi du 27 juillet 2005, et par conséquent, division des études supérieures en deux cycles d'enseignement $(3+2)$;

- concurrence des programmes de formation des NKJO ;

- nécessité de former les futurs enseignants à deux matières (suivant le modèle allemand) ;

- domination de l'anglais, et par conséquent, diminution du nombre d'écoles proposant l'enseignement du français, ce qui a causé une baisse d'intérêt pour nos études ;

- en réponse à cette situation, introduction de programmes parallèles de français pour débutants complets à l'université ;

- en raison des idées phares de l'UE - employabilité, internationalisation, pluridisciplinarité — nécessité de prévoir des programmes de formation préparant à des métiers concrets ;

- nécessité, par conséquent, de reformuler l'offre didactique des philologies pour proposer une formation où le français n'est plus qu'un élément de la formation professionnelle (par exemple, formation de traducteurs, formation pour les médias, formation pour l'édition, français dans les affaires, les philologies doubles, etc.) ;

- la loi de 2005 distinguant désormais aussi un troisième cycle d'études (doctorales), nécessité de prévoir un nouveau cadre pour la formation des futurs docteurs ;

- et pour le «monde des romanistes français », également un nouveau défi dû à l'intérêt croissant des candidats pour les études d'espagnol et d'italien : en quelque sorte, la concurrence des langues sœurs.

En réponse à ces défis «Plejada » a pris part activement dans les débats concernant l'avenir des philologies romanes :

- Avec la collaboration des collègues de République Tchèque, de Slovaquie et de Hongrie, notre association a organisé 21 écoles doctorales dans la zone géographique, ainsi que 3 rencontres post-doctorales pour assurer le suivi scientifique des jeunes chercheurs.

- ous ces événements ont été suivis de la publication de tomes thématiques ${ }^{13}$.

13 Toutes les publications de «Plejada » peuvent être consultées sur le site de la maison d'édition Oficyna Wydawnicza LEKSEM, Łask, série : Prace Naukowe Akademickiego Towarzystwa Romanistów Polskich «Plejada », <http://www.leksem.pl/>. 
- Avec le soutien du professeur José Kobielski, ancien conseiller universitaire, «Plejada » a lancé un grand débat qui a débouché sur le projet : Philologies romanes et orientation professionnelle des étudiants (2004). Dans le cadre de ce projet, une enquête a été effectuée auprès des anciens étudiants des philologies romanes pour en savoir plus sur leurs carrières professionnelles. En décembre 2004, un rapport de cette enquête a été préparé, suivi d'une rencontre, à Varsovie, des représentants de toutes les philologies romanes pour discuter des résultats et de la nouvelle forme de l'enseignement dans le cadre des études en deux cycles $(3+2)$. Enfin, en décembre 2005, "Plejada » a organisé à Varsovie une nouvelle rencontre des responsables des programmes des philologies romanes pour débattre de L'adaptation des contenus de l'enseignement de la philologie romane aux besoins professionnels des étudiants ${ }^{14}$.

- Dans toutes ces approches, répondant aux besoins du marché, «Plejada » n'a pas oublié la dimension scientifique. Ainsi, toujours avec le soutien de l'Ambassade de France en Pologne, un colloque post-doctoral intitulé Études françaises dans la société du XXI siècle. Défis et perspectives a été organisé en septembre 2010, à Wrocław ${ }^{15}$. Il a réuni de jeunes chercheurs de Pologne, République Tchèque, Slovaquie et Hongrie, et « a permis de confronter leurs pistes de recherche et leurs méthodologies, mais aussi de revoir et redéfinir le rôle des études françaises dans les sociétés européennes du $\mathrm{XXI}^{\mathrm{e}}$ siècle, avec leurs questionnements et leurs besoins ${ }^{16}$.

- L'association a aussi mené une enquête sur les conditions de la formation doctorale en Pologne et le déroulement des carrières des docteurs ayant soutenu leurs thèses entre 2000 et 2008. Sa synthèse a été présentée en 2008 par Halina Widła pendant l'Assemblée générale de « Plejada».

- Une autre enquête concernant les études de philologie romane dans les années 2009-2011 a été organisée par Teresa Jaroszewska. Ses résultats ont été présentés en 2011 sous la forme d'une brochure polycopiée.

Toute cette présentation montre comment la forme des études de philologie romane a été soumise à des changements politiques, économiques et sociaux à toutes les époques. Pourtant, il me semble que les romanistes de formation, peu importe leur spécialité par la suite, s'identifient toujours à leurs domaines de spécialité généraux qui sont la langue et la culture française et francophone, ce qui assure justement une certaine continuité.

14 Pour de plus amples informations, voir T. Tomaszkiewicz (dir.), Vers une nouvelle dimension des études romanes, Oficyna Wydawnicza LEKSEM, Łask 2006.

${ }^{15}$ Remarquons qu'au début de l'année 2010, on ne parle plus d'études romanes mais d'études françaises.

${ }^{16}$ T. Tomaszkiewicz, E. Skibińska, E. Biardzka, «Avant-propos », [dans :] Études françaises dans la société du XXI siècle. Défis et perspectives, E. Biardzka (dir.), Oficyna Wydawnicza LEKSEM, Łask 2011, p. 8. 


\section{UN DERNIER EXEMPLE CONTEMPORAIN}

La troisième génération de romanistes polonaises de ces 70 dernières années est celle de ma fille Idalia. Elle est née juste avant les transformations de 1989, et avec le soutien de ma mère, $\mathrm{j}$ 'ai voulu dès le début lui enseigner l'amour de la France et de la culture française. C'est ainsi qu'elle a fréquenté une école maternelle française, à Lyon, et que rapidement, j'ai malheureusement dû assister à sa rébellion lorsqu'elle a refusé toute communication en français dès ses 4 ans. Par la suite, nos déplacements familiaux ont voulu qu'elle devienne élève d'une école primaire allemande, à Bielefeld, à l'âge de 8 ans. Son exemple n'est peut-être pas commun, mais malgré tout assez fréquent vu la mobilité des Polonais dans toute l'Europe ces trente dernières années. Idalia a ensuite fait ses études secondaires au collège bilingue (polono-français) de Poznań, puis au lycée bilingue numéro I. Parallèlement elle a continué son apprentissage d'allemand et par conséquent en 2007, elle est devenue lauréate de deux Olympiades de langues, en français $\left(4^{\mathrm{e}}\right.$ place) et en allemand $\left(5^{\mathrm{e}}\right)$. Ce résultat lui a ouvert l'accès à différentes formes d'études, et elle a choisi l'Institut de linguistique appliquée de Varsovie. Elle y a terminé sa licence avec, comme première langue étrangère, le français, et a obtenu un diplôme de philologie avec spécialisation en linguistique appliquée (Licencjat na kierunku filologia, w specjalności: lingwistyka stosowana). Elle a poursuivi un deuxième cycle d'études d'interprétation à l'Université Adam Mickiewicz de Poznań. Le programme de ces études forme des interprètes en deux langues étrangères. Idalia a ainsi obtenu un diplôme de master de philologie avec spécialisation en philologies germanique et romane (Studia magisterskie na kierunku filologia, specjalność: filologia germańska z filologia romańska). Comme on peut le voir, les grands changements de programmes d'enseignement n'ont toujours pas appelé de changements de nomenclature des titres des diplômes.

Aujourd'hui, Idalia et ses camarades de promotion maîtrisent tous au moins deux langues étrangères et travaillent dans des entreprises internationales qui utilisent leurs compétences de polyglottes. Beaucoup d'entre eux se considèrent comme romanistes de vocation, même s'ils n'en font pas forcément leur métier.

\section{CONCLUSION}

Il est difficile de répondre à la question du présent volume de Romanica Wratislaviensia, qui est de savoir si nous assistons aujourd'hui à une rupture ou à une évolution de la philologie romane, s'il s'agit encore de romanica au sens traditionnel ou d'études romanes au sens moderne du terme. Pour moi, la question ne réside pas dans la nomenclature, mais dans l'objet d'investigation. Au moment de la fondation de la revue Romanica Wratislaviensia, il y a 50 ans, 
cette branche de la philologie se rapportait effectivement à l'étude des littératures et des langues issues du latin. D'ailleurs la langue et la culture latines étaient des matières obligatoires au programme de la formation des romanistes, des garantes, aussi, d'une identification européenne. Aujourd'hui, ces matières ont été en grande partie supprimées des programmes d'études pour laisser la place à d'autres, réputées plus pragmatiques. Toutefois, dans ce monde de plus en plus globalisé, il est probablement nécessaire de perpétuer une tradition, ce qui est d'ailleurs une des mission des universités à côté des défis du monde nouveau qu'elles doivent affronter. Ainsi, tout en nous soumettant aux attentes du marché contemporain, nous pouvons toujours défendre nos idées et nos vocations de romanistes. Paradoxalement, ce qui change le moins vite dans la vie universitaire, ce sont les noms et les appellations. Malgré tous les changements que je viens de décrire, nous continuons de délivrer aujourd'hui des diplômes de philologie romane, et non, par exemple, de traductologie ${ }^{17}$.

Les nombreuses revues de romanistes, en Pologne comme ailleurs, portent souvent cette identité inscrite dans leur nom. Il me semble qu'un changement de nom changerait également l'identité. Je serais donc très prudente et me garderais de prendre des décisions trop révolutionnaires en ce sens, car elles signifieraient que nous repartons de zéro. La force ne réside pas dans les ruptures mais dans les évolutions.

\title{
HOW PHILOLOGICAL VOCATIONS EVOLVE THROUGH GENERATIONS
}

\author{
Summary
}

In the study, the author discusses changes in the curricula and research in the field of traditionally defined Romance philology which have occurred in Poland over the last seventy years. The author's intention is to evidence that these changes have been evolutionary and remain in connection to the given historical context of the country, the economic and political transformations as well as the requirements of the job market. The study is illustrated with experiences from the professional lives of three generations of Romanicists: the author's, her mother's and her daughter's.

Key words: Romance philology, curricula, history, careers of the Romanicists.

${ }^{17}$ Pour une analyse plus détaillée de ce problème, voir l'article d'Elżbieta Skibińska, «Between Richness and 'Non-existence'. Polish Translation Researches as a Community », [dans :] Communities in Translation and Interpreting, K. Taivalkoski-Shilov, L. Tiittula, M. Koponen (dir.), Éditions québécoises de l'œuvre, Montréal 2014, pp. 253-274. 